\title{
SSC Reception over Kappa-Mu Shadowed Fading Channels in the Presence of Multiple Rayleigh Interferers
}

\author{
Aco Stevanovic ${ }^{1}$, Stefan Panic ${ }^{2}$, Petar Spalevic ${ }^{3}$, Bojan Prlincevic ${ }^{4}$, Milan Savic ${ }^{2}$ \\ ${ }^{1}$ Singidunum University, \\ Danijelova 32, Beograd, Serbia \\ ${ }^{2}$ Faculty of Natural Science and Mathematics, University of Pristina, \\ Lole Ribara 29, 38220 Kosovska Mitrovica, Serbia \\ ${ }^{3}$ Faculty of Technical Science, University of Pristina, \\ Kneza Milosa 7,38220 Kosovska Mitrovica, Serbia \\ ${ }^{4}$ Higher Technical Professional School in Zvecan, \\ St. Nusiceva No. 6, 38227 Zvecan, Serbia \\ b.prlincevic@vts-zvecan.edu.rs
}

\begin{abstract}
Modelling of complex wireless transmission scenario over fading channels in the presence of multiple cochannel (CCI) interferers will be presented in this paper. Propagation environment will be modelled with general kappamu shadowed model, which can be reduced to other wellknown model as its singularities. Probability density function (PDF) and cumulative distribution function (CDF) of resulting signal-to-interference (SIR) ratio statistics at the reception will be derived in the rapidly converging infinite-series expressions form. Obtained expressions will be further used for evaluation of standard wireless performance criterions, outage probability (OP) and average bit error rate (ABER) and their analysis in the function of transmission parameters. Finally, possible performance improvement will be considered for the case of possible application of switch-and-stay (SSC) diversity reception technique.
\end{abstract}

Index Terms-Wireless communication; Fading channels, Interference channels; Diversity reception; Bit error rate.

\section{INTRODUCTION}

One of major tasks in the process of designing wireless communication transmission link parameters (determining: level of transmitted power, applied modulation format, link distance, link capacity, pre-designated level of error at the reception, quality-of-service and grade-of-service at the reception, necessity of diversity appliance...) is to provide efficient mathematical model for evaluation of standard performance criterions of observed link, such are: outage probability (OP), average bit error rate (ABER) and channel capacity (CC) [1]. These models can be derived based on mathematical modelling of the unavoidable fading influence on both desired signal and total interfering signal. Fading influence arises as complex combination of influences of

Manuscript received 11 November, 2017; accepted 6 March, 2018.

This article was supported by the Serbian Ministry of Education, Science and Technological Development (projects III 44006 and TR32023). multipath (fast fading, short-time fading) and shadowing (slow fading, long-term fading). Multipath fading is a consequence of various phenomena, such are atmospheric ducting, ionosphere refraction, and reflection from various objects, causing that randomly delayed, reflected, scattered and diffracted signal components interact and could combine in constructive or destructive manner [2]-[5]. Multipath fading causes short-term signal envelope variations, and corresponding random instantaneous envelope values are statistically modelled by various models so far. Due to the shadowing from the objects in the propagation paths, the received signal is also affected by slow variations of the mean signal level with and its corresponding random instantaneous values are statistically modelled by various models so far [2].

Key aspect for the wireless communication systems analysis and simulation is accurate modelling and statistical characterization of corresponding faded channels. In order to incorporate simultaneous influence of multipath fading and shadowing, various models have been presented recently in the literature. However, a general model that provides best fit to real measurement scenarios, and has analytically traceable properties is $\kappa-\mu$ shadowed fading model presented in [6]. Presented model covers propagation scenarios with following physical interpretation: multipath fading, effects wireless signal in a line-of-sight (LOS) environment while shadowing is considered to affect only the power of the dominant components. This general fading model, written in the function of three parameters: shadowing fluctuation parameter $m$; clusters parameter $\mu$; and dominant components power/scattered waves power ratio parameter $\kappa$, can be simplified and transformed in other well-known fading models, by setting corresponding parameter values (i.e. One-sided Gaussian model: $\mu=0.5, \kappa \rightarrow 0, m \rightarrow 1$; Rayleigh model $\mu=1, \kappa \rightarrow 0, \mathrm{~m} \rightarrow 1$; Nakagami-m model, $\mu=m, \kappa \rightarrow 0, m \rightarrow 1$; Rician model, $\mu=1, \kappa=K, m \rightarrow 1$ 
$\kappa-\mu$ model, $\mu=\mu, \kappa=\kappa, m \rightarrow 1$; Rician shadowed model, $\mu=1, \kappa=K, \mathrm{~m}=m)$.

Wireless systems tend to conserve the existing disposable spectrum by reusing allocated frequency channels in areas that are geographically located as close to each other as possible, but unfortunately the amount of CCI that occurs determines constrains in distance for reusing wireless channels [7]. Total CCI signal is defined as the sum of multiple undesired interfering signals from other sources that have the same carrier frequency as the useful desired information signal. Sum of multiple equal average power interferers as main communication drawback assumption is suitable for two limiting cases that can bound the performances of any interference-limited systems: when interferers are on the cell edges closest to the desired user cell (worst interference case scenario) or where they are at the furthest edges (best interference case scenario) [8]. Efficient concept for upgrading system reception performances is space diversity concept, which assets on combining of two or more replicas of information bearing signal [2]. Based on complexity restriction put on the communication system and amount of channel state information available at the receiver several space diversity techniques could be realized. Switch-and-stay (SSC) diversity reception technique is the simplest technique for practical realization, among all space diversity reception techniques, since it processes only one of the received signal replicas at each time instant. In fading environment where the level of the CCI is sufficiently high as compared to the thermal noise (cellular systems), SSC selects and outputs a particular branch until its signal-to-interference (SIR) value falls below a predetermined threshold, when the combiner switches to another branch and stays there regardless of SIR of that branch, until next time instant. Diversity reception with multiple CCI over each fading channel was already observed in [9]. However, up to now, in the literature there is no consideration of this general kappa- mu shadowed fading model, when multiple interferers are also present. Major contribution of this paper is originality and wide scope of applicability of mathematical expressions that will be given bellow.

\section{SYSTEM MODEL}

Desired information signal with a $\kappa-\mu$ shadowed distributed random amplitude process can be presented by [6]

$$
\begin{aligned}
& f_{R}(R)= \frac{2 \mu_{d}^{\mu_{d}}\left(1+k_{d}\right)^{\mu_{d}} m_{d}^{m_{d}}}{\Gamma\left(\mu_{d}\right)\left(\mu_{d} k_{d}+m_{d}\right)^{m_{d}} \Omega_{d}^{\mu_{d}}} R^{2 \mu_{d}-1} \times \\
& \times \exp \left(\frac{-\mu_{d}\left(1+k_{d}\right)}{\Omega_{d}} R^{2}\right) \times \\
& \times{ }_{1} F_{1}\left(m_{d}, \mu_{d}, \frac{\mu_{d}^{2} k_{d}\left(1+k_{d}\right) R^{2}}{\left(\mu_{d} k_{d}+m_{d}\right) \Omega_{d}}\right),
\end{aligned}
$$

where $\Omega_{d}=E\left(R^{2}\right)$ denotes the average power of desired information signal, while $\mathrm{F}_{1}(a, b, \mathrm{z})$ and $\Gamma(a)$ denote the confluent hypergeometric function [10, Eq.(9.210/1)] and
Gamma function [10, Eq. (8.3107.1)], respectively. Physical interpretation of desired signal parameters, $m_{\mathrm{d}}, \mu_{\mathrm{d}}, \mathrm{k}_{\mathrm{d}}$ is given in [6].

Since we model each interfering signal with Rayleigh distribution, with PDF expressed as

$$
f_{I_{i}}\left(I_{i}\right)=\frac{2 I_{i}}{\Omega_{c i}} \exp \left(-\frac{I_{i}^{2}}{\Omega_{c i}}\right)
$$

The total PDF of the sum of $M$ Rayleigh distributed random variables (PDF of the amplitude of the total interfering signal)

$$
r=\sum_{i=1}^{M} I_{i}
$$

is then approximated by [9]

$$
p_{r}(r)=\frac{2 r^{2 M-1}}{\Omega_{c}^{M} \Gamma(M)} \exp \left(-M \frac{r}{\Omega_{c}}\right),
$$

where $\Omega_{\mathrm{c}}$ is the power of the resulting signal (resulting interferer from (2)) interference signal at the $i$-th branch of the diversity system, given in the function of the average power of each interferer $\Omega_{\mathrm{ci}}$ as

$$
\Omega_{c}=\frac{2 \Omega_{c i}}{M}\left(\frac{\Gamma(M+1 / 2)}{\Gamma(1 / 2)}\right)^{\frac{1}{M}}
$$

Since we are discussing an interference-limited system, in this case it can be shown that the instantaneous SIR, $\lambda=\mathrm{R}^{2} / \mathrm{r}^{2}$, at each branch, has the PDF of [1]

$$
f_{\lambda}(\lambda)=\frac{1}{\sqrt{\lambda}} \int_{0}^{+\infty} f_{R}(r \sqrt{\lambda}) f_{r}(r) r d r
$$

while average SIR, $S$, defined as $S=\Omega_{\mathrm{d}} / \Omega_{\mathrm{c}}$. hen by using the relation Equation (8.445) from [10], with respect to relation given with Equation (8.310.1) from [10] we derive

$$
\begin{gathered}
f_{\lambda}(\lambda)=\sum_{p=0}^{\infty} \frac{\mu_{d}^{\mu_{d}+2 p} m_{d}^{m_{d}} M^{M}\left(1+k_{d}\right)^{\mu_{d}+p} k_{d}^{p}}{\Gamma\left(\mu_{d}+p\right) p ! \Gamma\left(m_{d}\right) \Gamma(M)} \times \\
\times \frac{\Gamma\left(m_{d}+p\right) S^{M} \Gamma\left(\mu_{d}+M+p\right) \lambda^{\mu_{d}+p-1}}{\left(\mu_{d} k_{d}+m_{d}\right)^{m_{d}+p}\left(\mu_{d}\left(1+k_{d}\right) \lambda+M S\right)^{M+\mu_{d}+p}} .
\end{gathered}
$$

Capitalizing on (7), and by using the same mathematical transformations as in [11], closed-form expression for the cumulative distribution function (CDF) of the instantaneous SIR can be presented as

$$
\begin{gathered}
F_{\lambda}(\lambda)=\int_{0}^{\lambda} f_{\lambda}(t) d t=\sum_{p=0}^{\infty} \frac{\mu_{d}^{p} k_{d}^{p} m_{d}^{m_{d}} \Gamma\left(m_{d}+p\right) S^{M}}{\Gamma\left(\mu_{d}+p\right) p ! \Gamma\left(m_{d}\right) \Gamma(M)} \times \\
\times \frac{\Gamma\left(\mu_{d}+M+p\right)}{\left(\mu_{d} k_{d}+m_{d}\right)^{m_{d}+p}} \times
\end{gathered}
$$




$$
\times B_{z}\left(\mu_{d}+p, M, \frac{\mu_{d}\left(1+k_{d}\right) \lambda}{\mu_{d}\left(1+k_{d}\right) \lambda+M S}\right),
$$

with $\mathrm{B}_{\mathrm{z}}(a, \mathrm{~b}, \mathrm{z})$ being the incomplete Beta function [10, Eq. (8.391)]. Infinite-series expressions (7) and (8) converge rapidly, since about 20 terms should be summed in sum, to achieve accuracy at $5^{\text {th }}$ significant digit, for each combination of values of parameters $m_{\mathrm{d}}, k_{\mathrm{d}}, \mu_{\mathrm{d}}, M$ and $S$.

As already mentioned, in the interference-limited systems, SSC selects given branch until the SIR of that branch falls below a predetermined threshold, when SSC switches to another branch and stays there regardless of SIR value of that branch, so SSC output SIR can be expressed as

$$
f_{S S C}(\lambda)= \begin{cases}f_{\lambda_{1}}(\lambda) F_{\lambda_{2}}\left(z_{T}\right), & \lambda<z_{T}, \\ f_{\lambda_{1}}(\lambda)\left(1+F_{\lambda_{2}}\left(z_{T}\right)\right), & \lambda \geq z_{T} .\end{cases}
$$

Here, the predetermined switching threshold for the both input branches is denoted with $\mathrm{z}_{\mathrm{T}}$. Similary CDF of output SIR can be presented as

$$
\begin{gathered}
F_{S S C}(\lambda)= \\
=\left\{\begin{array}{lr}
F_{\lambda_{1}}(\lambda) F_{\lambda_{2}}\left(z_{T}\right), & \lambda<z_{T}, \\
F_{\lambda_{1}}(\lambda)-F_{\lambda_{2}}\left(z_{T}\right)+F_{\lambda_{1}}(\lambda) F_{\lambda_{2}}\left(z_{T}\right), & \lambda \geq z_{T} .
\end{array}\right.
\end{gathered}
$$

\section{PERFormance ANAlysis AND NumericAl RESUlts}

Outage probability (OP) is performance measure often used for determining the CCI level. This measure is crucial in helping the wireless communications system designers to meet the quality of service (QoS) and grade of service (GoS) demands [1]. In interference limited environment, OP is defined as the probability that the output SIR falls under defined protection ratio, which depends on applied modulation technique and expected QoS [2]

$$
P_{\text {out }}=\int_{0}^{x_{t h}} f_{S S C}(\lambda) d \lambda=F_{s S C}\left(x_{t h}\right) .
$$

Performance measure, which in best way describes the nature of the wireless communication system behaviour is the average bit error rate (ABER). ABER values are obtained capitalizing on conditional SER relations, which are conditioned over fading statistics which impairs the communication. Conditional SER are functions of the instantaneous SNR, and functional dependency is determined by the type of modulation scheme performed. Namely, when observing non-coherent binary modulation transmission, conditional SER is denoted with $\mathrm{P}_{\mathrm{e}}(\lambda)=1 / 2 \exp (-\lambda g)$. Then by averaging over instantaneous SIR values, $\lambda$, ABER can be obtained as [2]

$$
\begin{gathered}
\overline{P_{e}}=\int_{0}^{+\infty} f_{S S C}(\lambda) P_{e}(\lambda) d \lambda= \\
=\int_{0}^{z_{T}} f_{S S C}(\lambda) P_{e}(\lambda) d \lambda+\int_{z_{T}}^{+\infty} f_{S S C}(\lambda) P_{e}(\lambda) d \lambda,
\end{gathered}
$$

where $g$ denotes modulation constant, i.e., $g=1$ for Binary Differentially Phase Shift Keying (BDPSK) modulation and $g=1 / 2$ for the Binary Frequency Shift Keying (BFSK) modulation.

In Fig. 1, PDF of equivalent SIR random process has been presented for some values of signal parameters.

SSC output OP results for some values of system parameters is presented at Fig. 2, while ABER performance results obtained as a function of the average SIR's at the input branches of the balanced dual-branch SSC, $\left(S_{1}=S_{2}=\mathrm{z}_{\mathrm{T}}\right)$, for several values of $m_{\mathrm{d}}, k_{\mathrm{d}}, \mu_{\mathrm{d}}$, and number of interferers $M_{l}=M_{2}=M$ are presented at Fig. 3 and Fig. 4 .

First, we can see how the OP and ABER increase when the number of multiple independent co-channel interferers $M$ increases from 2 to 3 , due to growth of interference domination.

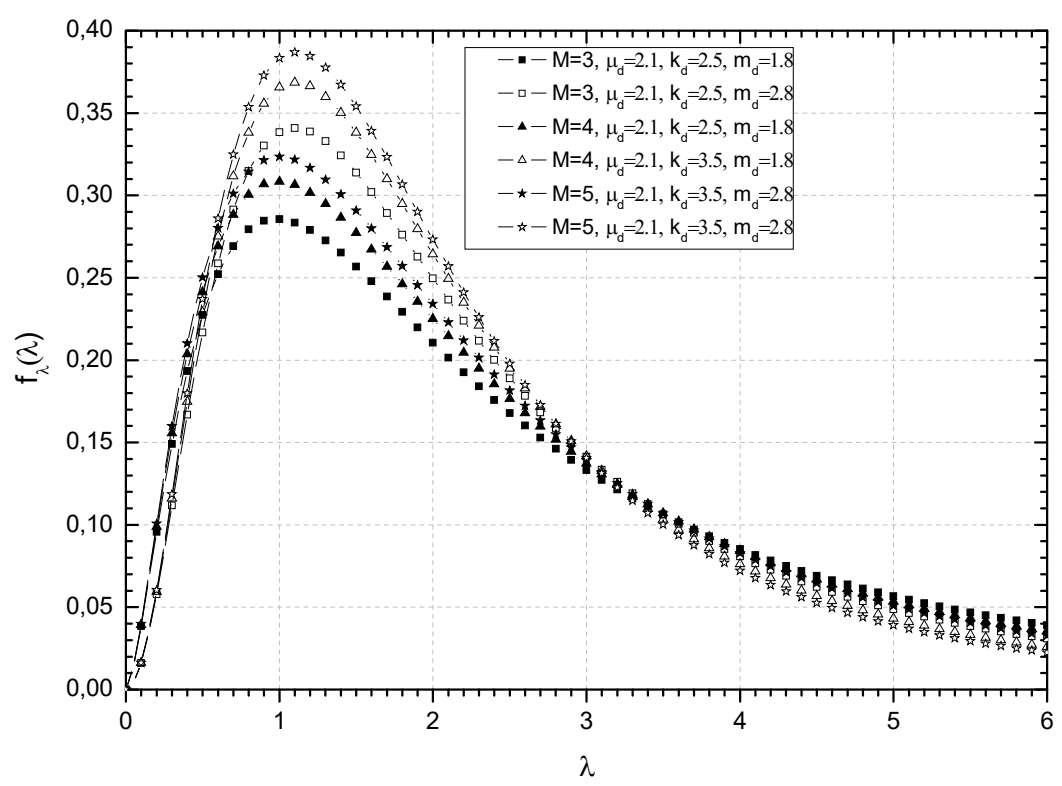

Fig. 1. PDF of SIR for various values of number of interferers. 


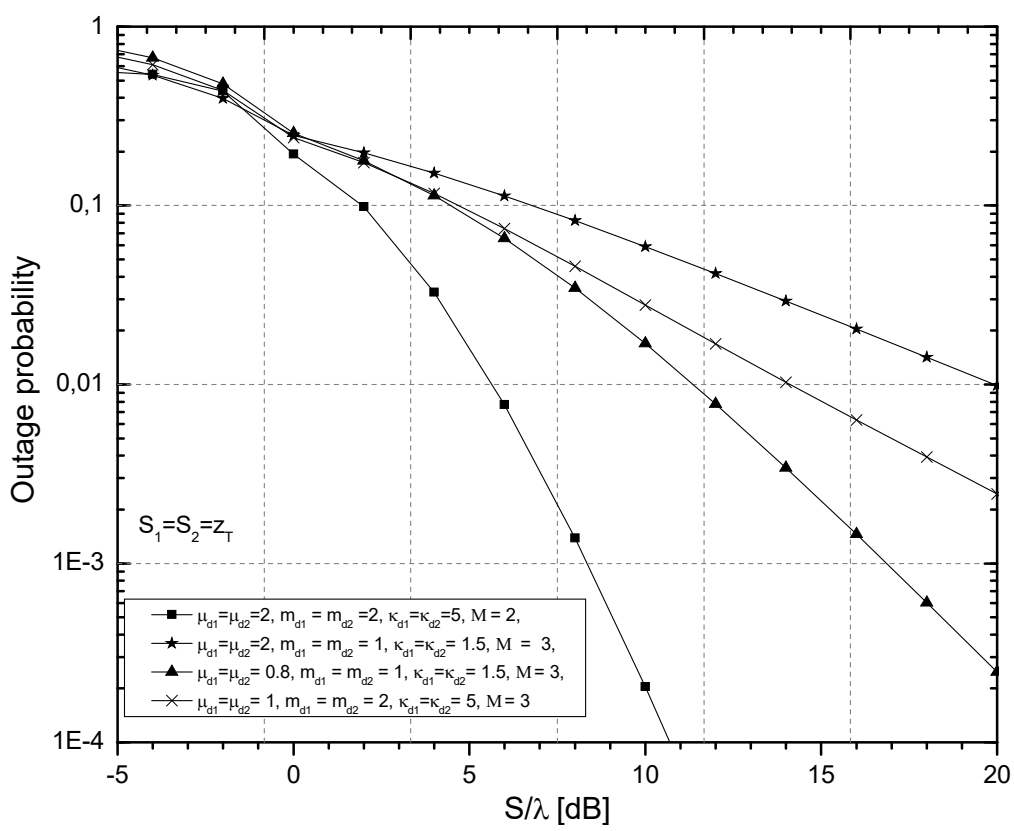

Fig. 2. SSC output OP for some values of system parameters.

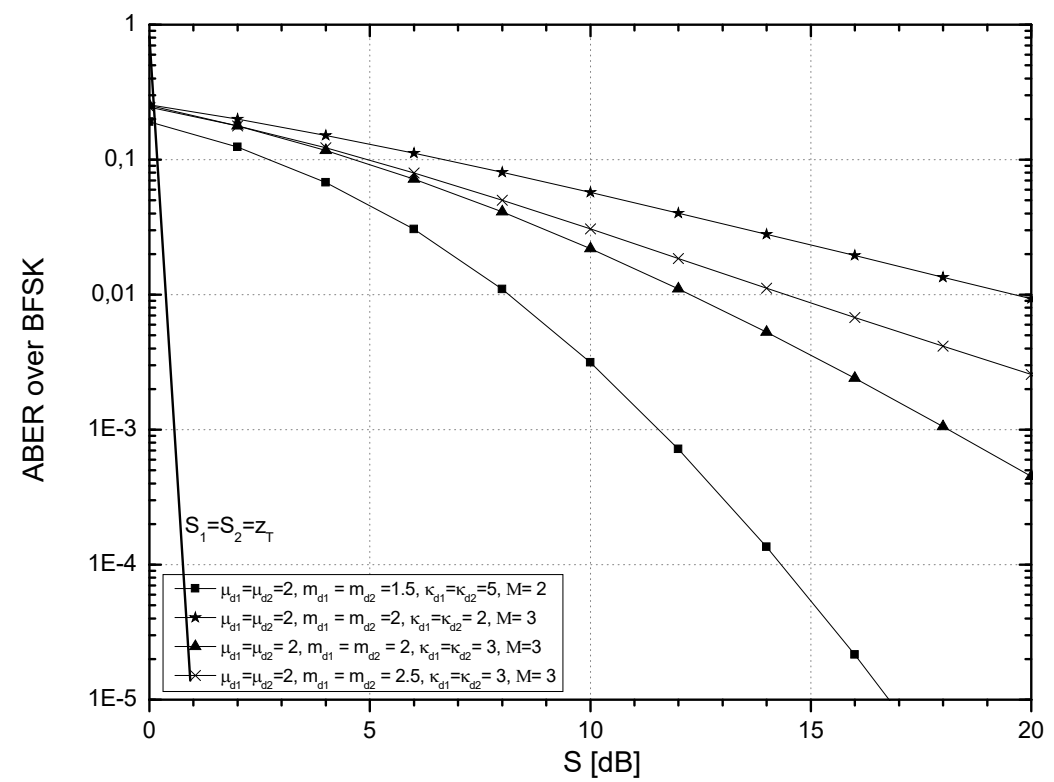

Fig. 3. SSC output ABER over BFSK modulation for some values of system parameters.

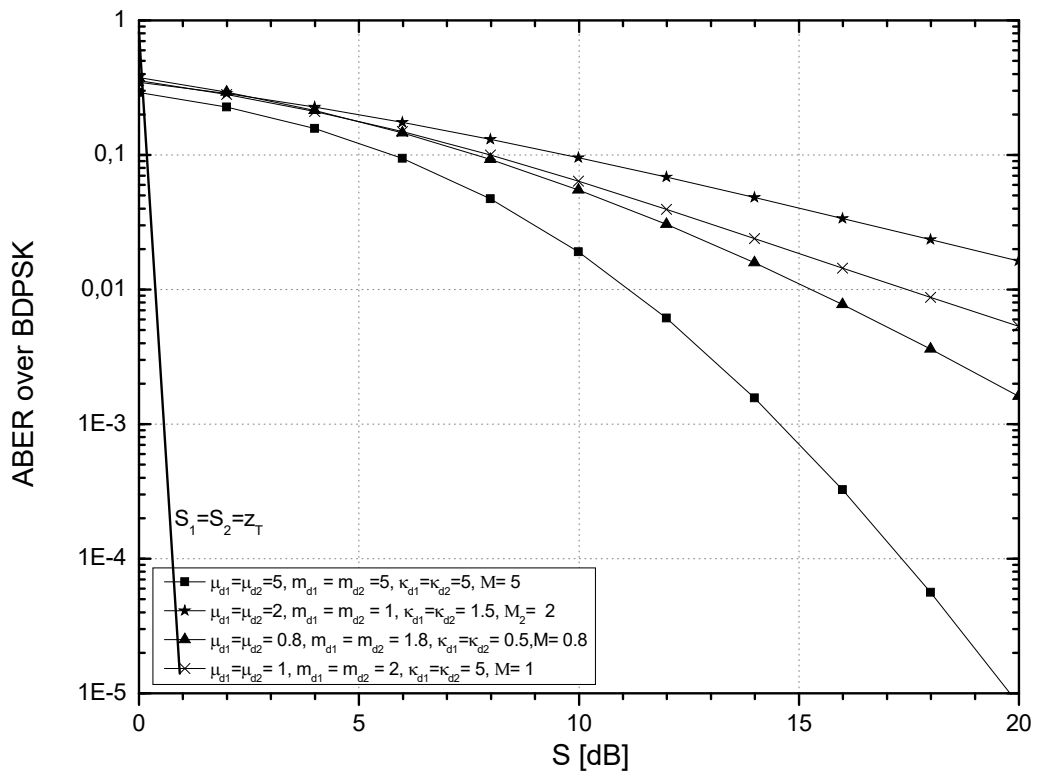

Fig. 4. SSC output ABER over BDPSK modulation for some values of system parameters. 
Among expected features, that performances improve (ABER and OP deteriorate) for higher values of $k_{d}$ and $m_{d}$, is interesting to observe how impact of $m_{d}$ is more notable for higher values of $k_{d}$.

Namely, in the cases when the dominant component of desired signal is very strong compared to its scattered components, the level of fluctuation due to shadowing in the dominant component can significantly affect system performances.

\section{CONCLUSIONS}

For the first time, characterization has been provided for equivalent SIR random process, which arises in wireless communication channel due to effects of kappa-mu shadowed fading and multiple Rayleigh CCIs, by deriving closed-form expressions for standard first order statistic measures, PDF and CDF. Further, SSC reception performances, $\mathrm{OP}$ and $\mathrm{ABEP}$, were efficiently evaluated and discussed in the function of system parameters. Among originality, wide scope of applicability of derived mathematical expressions is major contribution of the paper, since by setting corresponding parameters values, we can derive mathematical characterizations for various other SIR random processes that characterize concrete wireless propagation scenario phenomena.

\section{REFERENCES}

[1] M. Simon, M. Alouini, Digital Communication over Fading
Channels. USA: John Wiley and Sons, 2000. DOI: 10.1002/0471200697.

[2] S. Panic, M. Stefanovic, J. Anastasov, P. Spalevic, Fading and Interference Mitigation in Wireless Communications, USA: CRC Press, 2013. DOI: $10.1201 / \mathrm{b} 16275$.

[3] N. Kapucu, M. Bilim, I. Develi, "Performance of two-hop DS-CDMA systems using amplify-and-forward protocol over different fading channels", Elektronika ir Elektrotechnika, vol 22, no 1, pp. 97-101, 2016. DOI: 10.5755/j01.eee.22.1.14116.

[4] J. Bogucki, E. Wielowieyska, "Empirical season's fadings in radio communication at $6 \mathrm{GHz}$ band", Journal of telecommunications and information technology, no. 2, pp. 48-52, 2009.

[5] S. S. Ikki, S. Aissa, "Performance analysis of amplify-and-forward relaying over Weibull-fading channels with multiple antennas", IET Commun., vol. 6, no. 2, pp. 165-171, 2012. DOI: 10.1049/ietcom.2011.0264.

[6] J. Paris, "Statistical characterization of Kappa -Mu shadowed fading", IEEE T. Vehicular Technology, vol. 63, no. 2, pp. 518-526, 2014. DOI: 10.1109/TVT.2013.2281213.

[7] B. Lee, D. Park, H. Seo, Wireless Communications Resource Management, Willey, 2008.

[8] G. Cocco, M. Angelone, A. I. Perez-Neira, "Co-channel interference cancelation at the user terminal in multibeam satellite systems", International Journal of Satellite Communications and Networking vol. 35, no. 1, pp. 45-65, 2017. DOI: 10.1109/ASMSSPSC.2014.6934522.

[9] I. Petrovic, M. Stefanovic, P. Spalevic, S. Panic, D. Stefanovic, "Outage analysis of selection diversity over Rayleigh fading channels with multiple co - channel interferers", Telecommunication Systems Journal, vol. 52, no. 1, pp. 39-50, 2013. DOI: 10.1007/s11235-0119438-z.

[10] I. Gradshteyn, I. Ryzhik, Tables of Integrals, Series, and products, New York: Academic Press, 1980.

[11] S. Panic, M. Stefanovic, A. Mosic, "Performance analysis of selection combining diversity receiver overa $\alpha-\mu$ fading channels in the presemce of co-channel interference", IET Communications, vol. 3, no. 11, pp. 1769-1777, 2009. DOI: 10.1049/iet-com.2009.0023. 